\section{Next Generation Sequencing and Cancer}

\author{
Seyed Hashem Mir Mazloumi ${ }^{1 *}$ and Umaira Tabassum ${ }^{2}$ \\ ${ }^{1}$ Cytogenetic unit, Department of Pathology, Kidwai Memorial Institute of \\ Oncology, Bangalore, India \\ ${ }^{2}$ Pathology department, Kidwai Memorial Institute of Oncology, Bangalore, \\ India
}

\begin{abstract}
Genome instability and chromosomal aberrations are responsible for a number of genetic disorders and they are frequent features of cancer. Traditional cytogenetic methods for assessing chromosomal abnormalities like, FISH and karyotyping enable visualization of the genome, but often at low resolution. DNA microarray offers a simpler, more reliable, unbiased method that demonstrates higher resolution for assessing chromosomal aberrations. NGS can detect all aberration types including balanced translocations, inversions and sequence-level variations at least 100 -times more precisely down to the single nucleotide level. The advent of large data set has necessitated the need for development of computational tools to understand this data and interdisciplinary teams of scientists to formulate personalized approaches to the treatment of cancer and personalized cancer medicine.

Keywords: Cancer; Cytogenetic; Molecular cytogenetic; Sequencing
\end{abstract}

\section{Introduction}

Genome instability and chromosomal aberrations are responsible for a number of genetic disorders and they are frequent features of cancer. Cytogenetic analysis provides both numerical and structural abnormalities of chromosomes. The challenge of cytogenetic is simple yet tricky in practice. Cytogenomics primarily focuses on structural variability within chromosomes. Understanding chromosome variations through cytogenetic analysis is an integral part of current genomic medicine, playing a role in both constitutional disorders and cancer.

Traditional cytogenetic methods for assessing chromosomal abnormalities including, Fluorescent In-situ Hybridization (FISH) and karyotyping, enable visualization of the genome, but often at low

*Corresponding author: Seyed Hashem Mir Mazloumi, Cytogenetic unit, Department of Pathology, Kidwai Memorial Institute of Oncology, Bangalore, India, Tel: +91-9916994804; E-mail: khazarson@yahoo.com; khazarson@gmail.com

Citation: Mazloumi SHM, Tabassum U (2014) Next Generation Sequencing and Cancer. J Cancer Biol Treat 1: 002.

Received: July 19, 2014; Accepted: November 03, 2014; Published: November 17, 2014 resolution. Data generated from these cytogenetic analysis methods tend to be inconsistent and highly dependent upon the user's skill. In other way, for years, researchers have been limited by relatively crude tools at their disposal, such as Giemsa staining and FISH.

DNA microarrays offer a simpler, more reliable, unbiased method that demonstrates higher resolution for assessing chromosomal aberrations, but DNA sequencing techniques are boosting resolution to unprecedented levels, essentially bridge the gap between molecular cytogenetics and molecular genetics. Treatment decisions in oncology are increasingly informed by the results of molecular genetic testing [1].

\section{The Technologies of Cytogenetics}

\section{Conventional cytogenetic}

Chromosome staining and karyotyping: It's a technique using Giemsa to stain chromosomes and observe bands under microscope. It's great for looking at metaphase-scale chromosomal aberrations such as aneuploidy and gross rearrangements which actually can be seen under a microscope.

\section{Molecular cytogenetic}

\section{FISH}

It's a cytogenetic technique used to identify and localize the presence or absence of specific DNA sequence on a chromosome. Using FISH- based approaches, researchers can detect structural differences down to as small as 1-2 kbps.

\section{DNA microarrays}

The introduction of DNA microarrays allowed for querying of copy number variations, deletions and the like down to perhaps 5-10 $\mathrm{kb}$. Yet even these are unable to provide location-specific information about duplicate copies. They are limited to detection only sequences that are part of the references set used to design them.

\section{Next- Generation DNA Sequencing (NGS)}

NGS can detect all aberration types including balanced translocations, inversions and sequence-level variations at least 100-times more precisely down to the single nucleotide level. Clinicians have begun determining the tumor mutational status of multiple genes simultaneously using NGS.

NGS is well-suited to oncology testing because of its sensitivity. Important somatic mutation present in only a fraction of cell assayed can be reliably detected well below the levels detectable by traditional sequencing methods. Critically, the technology is also successful at generating high quality sequences data from formalin-fixed archived specimens, which are routinely available from standard pathology work- ups [2].

The most significant impact of NGS on cancer genomics has been the ability to re-sequence, analyze and compare the matched tumor and normal genomes of a single patient. With the significantly reduced cost of sequencing and tremendously enhanced throughout, it is now within the realm of possibility to sequence multiple patient samples of a given cancer type [3]. 
Multigene tests also make it easy to identify mutations known to be actionable in cancer types other than the one being tested. Such a finding can offer a treatment option when other options are exhausted. However, cases in which no mutation is found can also inform treatment decisions. Oncologists may rule out certain treatments with this information, potentially saving patients from toxicity and from time spent trying ineffective therapies.

\section{Clinical Implications of NGS}

Since its introduction in 2007, NGS has already made impressive advances in identifying cancer genes. The effectiveness of NGS in systemically identifying the spectrum of genetic alterations in cancer, including single- nucleotide variants, copy number variations, and large genomic rearrangements has opened the doors for sequencing all types of cancers, since then complete genomes of acute myeloid leukemia, breast cancer, lung cancer and some more have already been described. With this explosion of information using NGS, increasing numbers of cancer genomes, transcriptomes and methylomes become unrevealed. Integrating these data in practice, will help more accurate diagnosis, predict and monitor treatment outcome $[4,5]$.

\section{Examples of NGS in Medicine}

NGS has improved our understanding of the genetic pathology of the disease. This technique provides a highly sensitive method for simultaneous analysis of all monogenic diabetes genes. This single test can detect mutations previously identified by Sanger sequencing. The increased number of genes tested led to a higher mutation rate.

NGS is a useful application in disease treatment, screening and prenatal diagnosis. Prenatal diagnosis using fetal DNA in maternal serum represented the applicability of NGS in finding aneuploidies with non-invasive method.

The uses of NGS technology in human disorders like,

1.Monogenic disorders such as hearing loss, intellectual disabilities

2.Common diseases and complex disorders like cardiovascular disease, hypertension and obesity

3. Cancer had been studied.

The aim is to provide efficient and effective genetic data for best treatment; accurate, fast and cost-effective diagnosis of the patients which is a major concern in today's Genomic world $[6,7]$.

\section{Advantages and Limitations of NGS}

DNA sequencing is critical to understand and decode the genetic information for any biological system. Being cheaper and more advanced, NGS have shaped the new technical advances in different areas of cancer biology and treatment; moreover, massive sequencing platforms are more useful than other sequencing techniques. Several technical limitations of NGS can be including: quality control, data management and storage, reporting complex results and patent infringement that may affect laboratories using this technique $[8,9,10]$.

Revolution of NGS improved human health, although their expensive costs, ethical issues related to the produced genetic data and the need for user- friendly software in the analysis of the raw sequence have to be mentioned.

\section{Features of NGS}

The advent of large dataset has necessitated the need for development of computational tools to understand this data and interdisciplinary teams of scientists to formulate personalized approaches to the treatment of cancer and personalized cancer medicine. The hope is that a complete molecular understanding of cancer will provide the tools necessary to develop novel biomarkers for screening and drugs that target relevant cellular pathways [11].

In 2014 the joint meeting of the International Society for Cellular Oncology (ISCO) and the European Workshop on Cytogenetics and Molecular Genetics of Solid Tumors (EWCMST) discussed the ever increasing availability of new sequencing technologies which enabled the analysis of cancer genomes at increasingly greater details [12]. The challenge now is to relate structural changes in cancer genomes to the underlying disease mechanisms and to reveal opportunities for the design of novel therapies.

\section{References}

1. Kuleshov V, Xie D, Chen R, Pushkarev D, Ma Z, et al. (2014) Whole genome haplotyping using long reads and statistical methods. Nat Biotechnol 32: 261266.

2. Meldrum C, Doyle MA, Tohill RT (2011) Next generation sequencing for cancer Diagnostics: a practice perspective. Clin Biochem Rev 32: 177-195.

3. Daber R, Sukhadia S, Morrissette JJ (2013) Understanding the limitation of next generation sequencing informatics, an approach to clinical pipeline validation using artificial data sets. Cancer Genet 206: 441-448.

4. Sheun A, Foulkes WD (2010) Clinical implications of next generation sequencing for cancer medicine. 17: 39-42.

5. Hui $P$ (2012) Next generation sequencing: Chemistry, technology and applications. Top Curr Chem 10: 329-337.

6. Rabbani B, Tekin M, Mahdieh N (2014) The promise of whole-exome sequencing in medical genetics. J Hum Genet 59: 5-12.

7. Ellard S, Lango Allen H, De Franco E, Flanagan SE, Hysenaj G, et al. (2013) Improved genetic testing for monogenic diabetes using targeted next generation sequencing. Diabetologia 56: 1958-1963.

8. Hert DG, Fredlake CP, Barron AE (2008) Advantages and limitations of next generation sequencing technologies: a comparison of electrophoresis and non-electrophoresis methods. Electrophoresis 29: 4618-4626.

9. Hurd PJ, Nelson CJ (2009) Advantages of next generation sequencing versus the microarray in epigenetic research. Brief Funct Genomic Proteomic 8: $174-183$

10. Pavlopoulos GA, Oulas A, Lacucci E, Sifrim A, Moreau Y, et al. (2013) Unraveling genomic variation from next generation sequencing data. BioData Min 6: 13.

11. Gullapalli RR, Desai KV, Santana-Santos LK, Kant JA, Becich MJ (2014) Next generation sequencing in clinical medicine: Challenges and lessons for pathology and biomedical informatics. J Pathol Inform 3: 40.

12. Geurts van Kessel A (2014) The cancer genome: from structure to function. Cell Oncol 37: 155-165. 